\title{
Discussion on the Relationship Between Five Internal Organs Function and Circadian Rhythm
}

\author{
Zaoxia $\mathrm{Wu}^{1, *}$, Qiming Zhang ${ }^{2}$ \\ ${ }^{1}$ Shaanxi University of Traditional Chinese Medicine, Xianyang, Shaanxi 712046, China. \\ ${ }^{2}$ Medical Experimental Center, China Academy of Chinese Medical Sciences, Beijing 100700, China.
}

\begin{abstract}
How to cite this paper: Zaoxia $\mathrm{Wu}$, Qiming Zhang. (2021) Discussion on the Relationship Between Five Internal Organs Function and Circadian Rhythm. International Journal of Clinical and Experimental Medicine Research, 5(2), 219-224.
\end{abstract}

DOI: 10.26855/ijcemr.2021.04.017

Received: March 12, 2021

Accepted: April 10, 2021

Published: April 27, 2021

*Corresponding author: Zaoxia Wu, Shaanxi University of Traditional Chinese Medicine, Xianyang, Shaanxi 712046, China.

\begin{abstract}
As a new hot spot in modern medical research, circadian rhythm has formed a relatively complete biological theory system. In particular, the 2017 Nobel Prize in Physiology and Medicine was awarded, which affirmed the research of circadian rhythm. Traditional Chinese medicine classifies the functions of the human body as the functions of the Five Zang, Liufu, and Qiheng Houses, and discusses the circadian rhythm from the relationship between the physiological function changes of the viscera and the time. The spleen stores the function of transportation and transformation, the lung stores the respiratory function, the kidney stores the water and reproductive functions, the liver stores the regulation of relaxation and relaxation, and the heart stores the function of the spirit. The biological basis of the five-zang function is obvious. Circadian rhythm. Western medicine explores the circadian rhythm of the biological organism from the physiological basis and molecular mechanism. This article explores the phase distribution of the circadian rhythm of the five zang functions of traditional Chinese medicine from the biological basis of the five zang functions and the regulation mechanism of the circadian rhythm.
\end{abstract}

\section{Keywords}

Five-Zang Function, Executive Structure, Circadian Rhythm, Biological Clock, Biological Basis

Zang in Chinese medicine refers to the spleen, lungs, kidneys, liver, heart, gall bladder, stomach, large intestine, small intestine, triple Jiao, bladder, and six provinces in the body, the home of marrow, bone, pulse, gallbladder, and uterus [1]. The functions of the five Tibetans are as follows: the heart holds the main blood and the spirit, the body is combined with the pulse, the flower is in the face, the resuscitation is in the tongue, the will is the joy, the liquid is sweat, and the summer is in harmony; the spleen is the main movement and transformation, The main body controls the blood, the body unites the flesh, the limbs, the opening of the mouth, the flower is in the lips, the will is thought, the liquid is the salivation, and it is in harmony with the Changxia phase; the lungs are the main breath, the main water, Chaobaimai, attending knots, in the body to merge the skin, the hair is in the hair, the opening is in the nose, the throat is the gateway to the lungs, it is worry (sorrow) in the aspiration, and the tears in the liquid, which is in harmony with the autumn phase; the liver is the main control Ejaculation, the main storage of blood, in the body to combine the tendons, the flower is in the claws, the resuscitation is in the eyes, when the will is anger, when the liquid is tears, it is in harmony with the spring phase; it governs water and receives qi, combines bones in the body, and produces marrow. Its blooms are in hair, ears and yin in the orifice, fear in will, saliva in fluid, which is compatible with the winter phase [2].

In recent years, as a new hot spot in modern medical research, circadian rhythm has formed a relatively complete 
biological theory system. In particular, the 2017 Nobel Prize in Physiology and Medicine was awarded to Jeffrey C. Hall, Michael Rosbosh, and Michael W. Young praised his discovery of the molecular mechanism that controls the circadian rhythm, and the research on the biological mechanism of the circadian rhythm has achieved fruitful results [3]. Modern biomedicine believes that the circadian rhythm is an endogenous oscillating behavior and biological process of an organism with a cycle close to 24 hours. It is composed of endogenous circadian clock genes and can regulate sleep, exercise activity, blood pressure, body temperature and blood hormone levels, so that the organism Adapt to the changes of day and night.

\section{TCM's understanding of circadian rhythm}

"The Yellow Emperor's Internal Classic" systematically describes the influence of the day and night of the four seasons on the movement of human qi and blood and the relationship with diseases. For example, it puts forward the viewpoints of "vigor to the sky", "dirty qi method", and "correspondence between heaven and man", and mentions the physiology of the human body. The relationship between the occurrence and outcome of functions and diseases and the day and night changes in the environment. For example, "Dynasty will lead to the emergence of popularity, and the decline of sickness and Qi, so Dan Hui; the long in the day and the long, the victory of evil, and peace; the evening, the decline of popularity, the evil The beginning of life, so increase; in the night half of the people into the filth, evil spirits live alone, so nothing". "For those with yang qi, one day is the main thing, the yang qi grows in the daytime, the yang qi grows in the day, and the yang qi is declining in the west. The valve is closed" [4]. "Treatise on Febrile and Miscellaneous Diseases" describes the time-varying phenomenon of some diseases and puts forward the principle of timing treatment. For example, it is pointed out that the annual change of yin deficiency disease is "spring and summer drama, autumn and winter is bad"; the time rhythm of the recovery of six menstrual diseases is particularly detailed, pointing out that the cycle of six menstrual diseases recovery or transfer to another menstrual cycle is 6 to 7 days, and the time of remission of the six meridian diseases is also different; in terms of the appropriate time for sweating, vomiting, and proclamation, it is advocated that "sweat in spring and summer", "vomit in spring", and "get down in autumn" [5]. The "Ziwu Liuzhu Needle Jing” written by Yan Mingguang in the Jin Dynasty systematically discussed the theory and methods of Ziwu Liuzhu, and created the "Najia Method" of opening acupuncture points on time based on day-to-day dryness [6]. In "Compendium of Materia Medica", not only pointed out the importance of choosing medicine at the time, but also proposed choosing medicine at the time, noting that the growth of plants and the season of picking are related to the effect of medicine. Xu Lingtai, a famous doctor in the Qing Dynasty, pointed out in "Shen Ji Lu Yan", "Early and evening are not at the right time, not only useless, but also harmful" pointed out that the treatment of diseases should take into account the influence of time changes on the treatment, and conform to the circadian rhythm [7].

\section{The circadian rhythm of the five zang functions of traditional Chinese medicine}

"Suwen-Acquisition Law Time Theory" says: "Patients with liver disease, Pingdanhui, Xiaxian, very quiet at midnight; Heart disease, Huizhong at midnight, Pingdanjing; People with spleen disease, Huihui at daytime, strong at sunrise People with lung disease, Huihui under the sun, very quiet during the day, and quiet during the night; those with kidney disease, Huihui under the sun, very quiet in the four seasons, quiet under the sun. The main periods are as follows: the liver governs Pingdan, the heart governs the middle of the day, the spleen governs the sun, the lungs govern the Xiazhu, and the kidney governs the midnight" [8]. Modern medical research shows that it is feasible to understand the essence of TCM zombies at the cellular level or at the genetic level. For example, the functional characteristics of the kidney zombies are related to the growth, development, and repair of tissue cells; the functional characteristics of the liver zombies It is related to the regulation of the cell cycle and the polymerization and decomposition of proteins; the functional characteristics of the heart image are closely related to the post-transcriptional modification of the protein; the functional characteristics of the lung image are related to material transport and extra-membrane signal transduction [9].

\subsection{The biological basis of the five-zang function}

"Ziwu Liuzhu Needle Meridian" discusses the relationship between changes in the physiological functions of the five internal organs and time as follows: Chou Shi (1 o'clock to 3 o'clock), the liver meridian is the most prosperous. The liver hides blood. People's thinking and actions rely on the support of liver blood, waste blood needs to be eliminated, and fresh blood needs to be produced. This metabolism is usually completed when the liver meridian is most ugly. The theory of traditional Chinese medicine believes: "When a person lies down, the blood returns to the 
liver". At the time of Yin (3 o'clock to 5 o'clock), the lung meridian is the most prosperous. "Lung Chaobaimai”. After the liver pushes the blood out of the old when it is ugly, it provides fresh blood to the lungs and sends it to the whole body through the lungs. At this time (from 9 am to $11 \mathrm{am}$ ), the spleen meridian is most prosperous. "The spleen governs transportation and transformation, and the spleen governs the blood.” The spleen is the overall control of digestion, absorption, and excretion, and it is the commander of human blood. At noon (11 o'clock to 13 o'clock), the heart meridian is the most prosperous. "The heart governs the spirit, opens the tongue, and its blossoms are on the face.” The heart qi promotes the circulation of blood, nourishing the mind, qi, and muscles. At the unitary hour (17:00 to 19:00), the kidney meridian is the most prosperous. "The kidney stores the essence of reproduction and the essence of the internal organs. The kidney is the innate root.” The human body goes through the Shenshi to vent fire and detoxification, and the kidney enters the stage of storing the essence when it is unitary [10].

The main function of spleen storage is to digest food, absorb nutrients, detoxify, and excrete feces. Its biological basis includes the digestive tract (mouth, pharynx, esophagus, stomach, large and small intestine), skeletal muscle (chewing and swallowing, defecation), glands (salivary glands, gastric exocrine glands, pyloric glands, duodenal glands, Small intestine glands), digestive enzymes, etc. The respiratory function of the lung reservoir refers to the function of the respiratory system inhaling $\mathrm{O}_{2}$ and exhaling $\mathrm{CO}_{2}$, red blood cells and plasma carry $\mathrm{O}_{2}$ and $\mathrm{CO}_{2}$, and regulate the balance of acidolysis. Its executive structure consists of the respiratory tract (nose, larynx, trachea, bronchus) and participation Respiratory smooth muscle and skeletal muscle, vocal cords, resonance cavity and other components, including connective tissue, blood vessels, lymphatic vessels, lymph nodes and nerves. The gasification function of the kidney reservoir refers to the assimilation and dissimilation system to synthesize and decompose organic matter, and produce, carry and discharge energy. It includes organelles and enzymes responsible for the synthesis and decomposition of organic matter, body fluids and ATP responsible for energy carrying, and heat generating radiator. The process in which macromolecular substances (such as sugar, fat, and protein) in cells are broken down into metabolic end products (such as $\mathrm{CO}_{2}$, uric acid, and urea) and release energy. The excretory function of the liver is the function of the visceral nervous system to produce and transmit visceral sensory and motor signals to coordinate visceral (digestion, breathing, urinary, reproductive organs), cardiovascular and glandular activities, and the limbic system to produce emotions. The peripheral processes of cranial ganglion (including knee ganglion, inferior ganglion of glossopharyngeal nerve, and inferior vagus nerve) cells are distributed in internal organs (eyes, lacrimal gland, parotid gland, sublingual gland, submandibular gland, etc.) along with facial nerve, glossopharyngeal nerve, and vagus nerve. Surface blood vessels, heart, larynx, trachea, lung, stomach, liver, gallbladder, pancreas, small intestine, large intestine, adrenal gland, kidney, bladder, genitals, the central process enters the brainstem along with the facial nerve, glossopharyngeal nerve, and vagus nerve, and finally a solitary bundle. The transmission pathway of nuclear and visceral movement signals, including smell, taste, etc. The central nervous system produces mental activity, the most obvious manifestation is the sleep-wake rhythm [11].

\section{Circadian rhythm regulation system}

\subsection{Molecular regulation mechanism of circadian rhythm}

In mammals, the normal physiological structure of the clock is constructed by the central and surrounding clocks. It is a hierarchical network structure with a transcription-translation feedback loop as the core. The circadian rhythm is an endogenous, gene-based biological rhythm, whose core is the positive arm formed by the basic helix-loop-helix (PAS) transcription factor BMAL1 and the circadian movement output cycle protein (CLOCK) heterodimer, and Period (PER) and Cryptochrome (CRY) heterodimer formed a feedback loop formed by the negative arm [12]. The first negative feedback loop composed of PER/TIM and CLK/CYC explained the circadian rhythm of per and tim mRNA; the second feedback loop identified vrille (vri) and Pdp1 $1 \varepsilon / \delta$ (PAR domain protein $1 \varepsilon, \delta$ ). The protein levels of the two transcription factors present a circadian rhythm, and the clk they regulate also present a circadian rhythm; the third negative feedback loop is composed of CLK/CYC and the transcription repressor CWO (CLOCKWORK ORANGE) containing the bHLH-orange domain, Composition [13].

The periodic oscillation of the circadian rhythm depends on the Transcription-Translation Oscillation Loop (TTO). TTO is composed of a variety of core biological clock genes and clock-control genes, including the positive components CLOCK and BMAL1 in the cell, the negative components CRY and PER, and the auxiliary loop composed of ROR $\alpha$ and REV-ERB $\alpha$. Among them, transcription factors CLOCK and BMAL1 form a heterodimer, which binds to the circadian clock genes per and cry, thereby activating the transcription of target genes; when the expression of PER and CRY series proteins accumulate to a certain extent, they are transferred from the cytoplasm to the nucleus, and the PER/CRY heterodimer. The aggregate form acts as a negative regulator and directly interacts 
with CLOCK/BMAL1 to inhibit its transcriptional activity. PER and CRY proteins can be further degraded after being phosphorylated and ubiquitinated, so that their inhibitory effect on CLOCK/BMAL1 is lifted, and Restart the signal transmission process of the TTO loop; the CLOCK/BMAL1 heterodimer activates the transcription of per and cry series genes, and can also induce the expression of nuclear receptors REV-ERB $\alpha$ and ROR $\alpha$.

Because the above-mentioned gene transcription and protein nucleus reactions take a certain time to complete, the changes in the up- and down-regulation of the expression of these core biological rhythm-regulating genes are maintained at the oscillation period of about $24 \mathrm{~h}$. This molecular oscillation not only makes the transcriptional activity of the CLOCK/BMAL1 heterodimer present rhythmic characteristics, but also makes the downstream clock-controlled genes regulated and controlled by the above core biological clock genes be rhythmically expressed, so that the biological rhythm signals can be output. The TTO loop shows the core role of the transcription and translation mechanism in the generation and maintenance of the circadian rhythm. At the same time, the post-translational modification (phosphorylation/dephosphorylation, acetylation/deacetylation, etc.) and degradation (ubiquitin) of various clock proteins (Chemical/proteasome diameter), can realize the fine-tuning of the TTO loop (such as adjusting the expression phase and oscillation period), so it can also play an important role in the periodic cycle of the circadian rhythm [14].

The composition of the feedback loop, through the regulation of transcription, translation, and post-translational levels, multi-level regulation leads to circadian fluctuations in the mRNA and protein levels of the core members of the biological clock, which provides a molecular basis for the 24-hour rhythm.

\subsection{Physiological basis of circadian rhythm}

The central and peripheral biological clock system, rhythm input system and rhythm output system constitute the physiological basis of the circadian rhythm. Among them, the central biological clock of mammals is located in the anterior hypothalamic suprachiasmatic nucleus (SCN). As the main pacemaker of the biological clock system, it plays a leading role in the generation and regulation of circadian rhythms. In addition to SCN, the amygdala and hippocampus of the brain. There are also expressions of biological clocks in parts such as olfactory bulbs and olfactory bulbs, which play an important regulatory role in maintaining neural activities in their respective regions [15]. Peripheral circadian clocks exist in the heart, liver, spleen, kidneys and other tissues. They are used as the secondary pacemaker of the circadian clock system. On the one hand, they are directly or indirectly regulated and controlled by various nerves, body fluids and other signal factors under the regulation of the central circadian clock; On the one hand, it can operate independently of the control of SCN, regulating the expression of genes related to the specific functions of each tissue [15]. The input system of circadian rhythm is composed of melanopsin, retinal hypothalamic tract (RHT) and SCN, and regulates the expression of central circadian clock genes through changes in light signals. The output system of the circadian rhythm includes the neuroendocrine system and the autonomic nervous system. The oscillating signal generated after the light signal is transmitted to the SCN area through the RHT is transmitted to other parts of the brain through the nerve fiber to control the autonomic nervous system and the hypothalamic-pituitary axis. The activities of the endocrine system and the sleep/wake-up system produce the circadian rhythm of life activities on the overall level [16].

\section{The relationship between the five-zang functions of traditional Chinese medicine and the circadian rhythm}

The original phase refers to the value of the variable at each time point of the cycle. In time biology, it refers to the time point at which a certain characteristic value of the physiological variable emerges in the rhythm cycle, also known as the phase. The peak phase and the valley phase are the main signs of the rhythmic phase characteristics. They are the time positions of the peak and valley in the rhythm cycle, respectively. If a certain moment is used as the time reference point of the rhythm cycle, and $360^{\circ}$ represents a cycle, the angle between the time point at which the peaks and valleys appear and the time reference point is often used to represent the peak phase and the valley phase. For example, the peak value of the circadian rhythm of human body temperature usually appears at 15:00. If 00:00 is the time reference point of the circadian rhythm cycle, the peak phase is $225^{0}$ [17].

The time at which a certain value of a periodic variable (such as peak or valley) appears (that is, its phase) is earlier or later than usual, which is called phase shift. The one that appears early is called phase forward, or the phase is left; the one that appears delayed is called phase backward or phase right. Due to the change in frequency, the phase of a rhythm is delayed or lags behind the other rhythm. Phase is called phase lag. When the phase of the rhythm changes by $180^{\circ}$, it is called rhythm inversion, also called rhythm inversion or rhythm inversion. If the pe- 
riods of the two rhythms are the same, but their phases are always different by $180^{\circ} \pm 15^{0}$, you are the opposite phase of the two rhythms [18-22].

Traditional Chinese medicine believes that the functions of spleen storage, lung storage, kidney storage, liver storage, and heart storage are respectively prosperous in Changxia (the third month of summer), autumn, winter, spring, and summer. Summer, autumn, winter, spring, and summer are uniquely affected by the climate, such as long and humid summers, the spleen storage and transportation functions are easily affected and loss of appetite (commonly known as bitter summer); autumn is dry, the lung storage function is easily affected and coughing and wheezing. In the cold winter, the function of kidney gasification is easily affected and painful; the spring is windy, the function of blood storage in the liver is easily affected and the limbs tremble; the summer is hot, the function of the heart and the soul is easily affected and heatstroke. The peak phase distribution of the circadian rhythm of the five-zang function is: the spleen is stored at 14:00-15:00, the lungs are stored at 20:00-21:00, the kidneys are stored at 02:00-03:00, and the liver is stored at 02:00-03:00. From 08:00 to 09:00, Xin Zang Sheng is in 14:00 to 15:00.

\section{References}

[1] Li Ruhui. (2002). Discussion on Several Theoretical Issues of "Basic Theory of Traditional Chinese Medicine” (I)—Zang Xiang (Zang Fu), Zang Xiang (Tiang Mansion) [J]. Journal of Zhejiang College of Traditional Chinese Medicine, 2002, 26(4): 65-66.

[2] Sun Guangren. (2002). Basic theories of traditional Chinese medicine [M]. 7th edition. Beijing: China Press of Traditional Chinese Medicine, 2002: 84-8.

[3] Yuan Li, Li Yirou, Xu Xiaodong. (2018). Time Biology-Nobel Physiology in 2017 Or interpretation of medical awards [J]. Genetics, 2018, 40(01): 1-11.

[4] Li Jialin, Ren Xiaoqiao, Wu Jiajie, Zheng Lijuan, Li Longmei, Deng Zhiyun. (2019). Theoretical characteristics of the circadian rhythm in the Yellow Emperor's Internal Classic [J]. Chinese Journal of Traditional Chinese Medicine, 2019, 37(01): 89-91.

[5] Chen Yangzi, Wang Shunmei, Sun Yuanmei, Han Xiaoxue, Yang Wenting, Li Xiaojun. (2009). “Yellow Emperor’s Internal Classic" and "Treatise on Febrile and Miscellaneous Diseases" “Take Measures to Conditions" Thoughts [J]. Chinese Journal of Traditional Chinese Medicine, 2009, 24(S1): 89-92.

[6] Gao Shaocai. (2008). Comparative study of Ziwu Liuzhu and Time Medicine [J]. Modern Distance Education of Chinese Medicine, 2008(11): 1307-1309.

[7] Zhang Binglun. (1981). The understanding and utilization of animal circadian rhythms in ancient China [J]. Acta Zoology, 1981(01): 98-105.

[8] Zhang Liping. (2016). “Huangdi Neijing” understanding of the human body’s daily rhythm [J]. Chinese Journal of Basic Medicine in Traditional Chinese Medicine, 2016, 22(10): 1288-1290.

[9] Li Yanying. (2015). Exploring the essence of traditional Chinese medicine from the circadian rhythm of cell function. Beijing: Beijing University of Traditional Chinese Medicine, 2015.

[10] Lecture Hall. (1991). Ziwu Liuzhu Shuoao [M]. Beijing: People’s Medical Publishing House, 1991.

[11] Zhang Qiming, Liu Baoyan. (2014). The function positioning map of the five zang system of traditional Chinese medicine [M]. Beijing: People's Health Publishing Society, 2014: 8, 11.

[12] An Yang, Xu Ying. (2015). Research progress on the mechanism of mammalian circadian rhythm [J]. Life Science, 2015, 27(11): 1372-1379.

[13] Zhang Luoying, Ye Xiaoxue. (2015). Regulation mechanism of Drosophila circadian rhythm [J]. Life Science, 2015, 27(11): 1345-1354.

[14] The regulatory system for the generation and maintenance of circadian rhythm [M]. Beijing: People’s Medical Publishing House, 1991.

[15] Tian Ye, Su Gang, Liu Jifei, Liu Xiaoyan, Zhang Zhenchang. (2020). Influencing factors of rodent ischemic cerebral infarction model: circadian rhythm [J]. Journal of Lanzhou University (Medical Edition), 2020, 46(03): 63-68.

[16] Saper, C. B., Scammell, T. E., Lu, J. (2005). Hypothalamic regulation of sleep and circadian rhythms [J]. Nature, 2005, 437(7063): 1257-1263.

[17] Deguchi, T. (1979). Circadian rhythm of serotonin N-acetyl-transferase activity in organ culture of chicken pineal gland. Science (New York) 1979, 203: 1245.

[18] Honma, S., et al. (1984). Effects of elimination of maternal circadian rhythms during pregnancy on the postnatal development of circadian corticosterone rhythm in blinded infantile rats. Endocrinology, 1984, 114: 44-50.

[19] Wever, R. A. (1979). Results of experiments under temporal isolation. In: The Circadian System of Man. Heidelberg; Springer, 1979. 
[20] Fan Qingduan, Liu Zengrong. (2017). Phase synchronization to discuss mammalian circadian rhythms [J]. Journal of Shanghai University (Natural Science Edition), 2017, 23(02): 290-297.

[21] Aschoff, J., et al. (1969). Circadiane Periodik des Menschen unterdem Einfluss von Licht- Dunkel- Wechseln unterschiedlicher periode. Pflugers Arch., 1969, 306: 58.

[22] Inouye, S., TKawamura, H. (1982). Characteristics of a circadian pacemaker in the suprachiasmatic nucleus. J Comp Physiol., 1982, 146: 153. 